\title{
A review of umbilical venous catheter-related complications at a tertiary neonatal unit in Singapore
}

\author{
Sharon Si Min $\underline{G o h}^{1}$, MBBS, Sheau Yun $\underline{K a n}^{1}$, MBBS, MMed, Srabani Bharadwaj ${ }^{1}$, MRCPCH, FRCPCH, \\ Woei Bing $\underline{\text { PoOn }}^{1}$, MRCPCH, FAMS
}

INTRODUCTION: Umbilical venous catheters (UVCs), commonly inserted in neonates for vascular access, are not without complications.

METHODS: A single-centre retrospective cohort study that reviewed complications related to UVC insertion in neonates was conducted in a tertiary neonatal unit in Singapore from January 2016 to July 2017. Ideal UVC position was defined as catheter tip within $0.5 \mathrm{~cm}$ above or below the diaphragm. Catheter-related sepsis was defined as clinical or biochemical abnormalities suggesting any new-onset or worsening sepsis 72 hours before or after removal of UVCs, with or without positive culture. Catheter-associated bloodstream infection (CABSI) was defined as positive microbiological growth in one or more blood cultures obtained from a symptomatic infant up to two days after UVC placement or within 48 hours of catheter removal.

RESULTS: 108 patients had UVC insertions. Mean gestational age and birth weight were $30.4 \pm 4.0$ weeks and $1,536.2 \mathrm{~g}$ \pm 788.9 g, respectively. Mean UVC duration was 6.6 days. The UVC was in an ideal position in $27(25.0 \%)$, deep in 13 $(12.0 \%)$ and short in $35(32.4 \%)$ neonates. One-third of the UVCs $(n=33)$ were malpositioned. Catheter-related sepsis was observed in $16(14.8 \%)$ neonates, with 5 (4.6\%) having CABSI. The most common organism was coagulase-negative Staphylococcus. Other complications include peritoneal extravasation in $3(2.8 \%)$ patients, with two requiring surgical intervention. Venous thrombosis occurred in $2(1.9 \%)$ neonates and was managed conservatively.

CONCLUSION: Although complication rates were in line with international norms, UVCs were associated with serious complications and should be judiciously used.

Keywords: complications, newborn, umbilical venous catheter

\section{INTRODUCTION}

Umbilical vein catheterisation is a commonly performed invasive procedure in neonates. ${ }^{(1)}$ It serves as an immediate postnatal access for intravenous fluids or emergency medications, as well as for the administration of hypertonic fluids such as total parenteral nutrition. They provide painless and quick vascular access immediately after birth in high-risk preterm neonates.

The use of umbilical venous catheters (UVCs) has been associated with multiple complications, including catheterassociated bloodstream infections (CABSIs), ${ }^{(2,3)}$ venous thrombosis, ${ }^{(4)}$ and hepatic ${ }^{(5)}$ and cardiac $^{(6,7)}$ complications, although the relative incidence is not well established. ${ }^{(8)}$ Proper catheter position is important to avoid the development of severe complications.

In 2014, two deaths related to UVC extravasation were notified to the British Association of Perinatal Medicine and National Health Service National Reporting and Learning System. Both involved parenteral nutrition infusion via low-lying UVCs. These cases prompted a national survey of practices in the United Kingdom, which was published in $2015^{\left({ }^{(8)}\right.}$ and the current audit. To our knowledge, no other prior local figures were available on UVC practices and complication rates. Indeed, there is great heterogeneity in the practice of UVC among different neonatal units and a paucity of published literature regarding its complications internationally.

This study aimed to evaluate the complications associated with UVC use, its risk factors for complications, and whether UVC positions are associated with these complications. To the best of our knowledge, this was the first such review of UVC use and its complications in Singapore. We presumed that our findings would help to inform clinicians of the risks and benefits of UVCs, especially in preterm infants.

\section{METHODS}

This was a retrospective review of a cohort of neonates admitted from January 2016 to July 2017 at Singapore General Hospital, Singapore, with UVC insertion. We collected and analysed the baseline demographics, duration of UVC use, day of insertion, position of catheters and complications associated with the use of UVCs.

Data collected included baseline demographics (gestational age, birth weight and gender), UVC tip positions and complications observed. Birth weight was further classified as small, appropriate or large for gestational age based on the cut-off percentiles $<10 \%$, $10 \%-90 \%$ and $>90 \%$, respectively, according to the genderspecific Fenton growth chart for preterm infants.

UVC tip positions were categorised as deep, ideal, short or malpositioned. An ideal position was a UVC tip at the level of the diaphragm, seen on an anteroposterior abdominal radiograph, with allowance for up to $0.5 \mathrm{~cm}$ above or below the level of the diaphragm. A deep UVC tip was when it was placed more than $0.5 \mathrm{~cm}$ above the level of the diaphragm, while a low-lying UVC was when it was placed more than $0.5 \mathrm{~cm}$ below the level of

${ }_{1}^{1}$ Department of Neonatal and Developmental Medicine, Singapore General Hospital, Singapore

Correspondence: Dr Sharon Goh Si Min, Medical Officer, Department of Neonatal and Developmental Medicine, Singapore General Hospital, Outram Road, Singapore 169608. Sharon.goh@mohh.com.sg 
the diaphragm. Malpositioned catheters were those that turned laterally into the hepatic circulation. Complications reviewed included catheter-related sepsis (including CABSI), extravasation, venous thrombosis and death.

Catheter-related sepsis was defined as clinical, haematological (full blood count and immature-to-total neutrophil ratio) or infective marker (C-reactive protein) abnormalities to suggest any new-onset or worsening sepsis 72 hours before or after the removal of a UVC with or without a positive culture. CABSI was defined as the presence of bacteria or fungus in one or more blood cultures obtained from a symptomatic infant after two days of placement of a central catheter or within a 48-hour period after catheter removal. ${ }^{(9)}$ We adopted this definition from the study by Shalabi et $\mathrm{al}^{(9)}$ from the Canadian Neonatal Network, as similar to their rationale, the need for two blood cultures or a blood culture to be drawn from the catheter for diagnosis of catheter-related bloodstream infection (CRBSI) was not pragmatic for neonatal practice. ${ }^{(10)}$

The demographics of infants were shown using descriptive statistical methods and presented as mean and standard deviation. CABSI rate was calculated using the above definition of CABSI rate per 1,000 catheter days.

\section{RESULTS}

From January 2016 to July 2017, there were 108 neonates with a UVC inserted. Mean gestational age was $30.4 \pm 4.0$ weeks, while mean birth weight was $1,536.2 \mathrm{~g} \pm 788.9 \mathrm{~g}$. Table I shows the baseline demographics of the neonates.

The mean UVC duration was $6.6 \pm 2.7$ (range 1-18) days. Overall, 33 (30.6\%) UVCs were malpositioned, 27 (25.0\%) were ideal, 13 (2.0\%) were deep and 35 (32.4\%) were low-lying. Among the 108 neonates, 16 (14.8\%) had catheter-related sepsis and of these, 15 neonates required a change in the intravenous antibiotics that were chosen for treatment.

A total of $5(4.6 \%)$ patients had CABSI, which translated to a CABSI rate of 7.0 per 1,000 catheter days. The most common organism was coagulase-negative Staphylococcus (CONS; n = 4), while the last neonate grew Enterobacter cloacae (E. cloacae). Among the patients with CABSI, four had further positive umbilical line tip cultures, although these were not routinely tested for all patients. Three tip cultures grew CONS, while one grew E. cloacae. Table II summarises the clinical presentation and culture results of neonates with CABSI. One of these patients subsequently died from unrelated sepsis.

In all, $3(2.8 \%)$ neonates had UVCs complicated by extravasation. All three patients were premature at 24 weeks, 31 weeks and 34 weeks, with birth weights of $615 \mathrm{~g}, 1,660 \mathrm{~g}$ and 2,140 g, respectively. Two neonates had UVCs inserted on Day 1 of life and one had the UVC inserted on Day 2 of life. Two UVCs were low-lying, while one was malpositioned into the liver. All had parenteral nutrition infused through the UVCs. These neonates were noted to have abdominal distension that was associated with metabolic derangements, namely metabolic acidosis and hyponatraemia, which developed within 3-7 days after UVC insertion. All underwent abdominal radiography and
Table I. Baseline demographics of neonates $(n=108)$.

\begin{tabular}{|ll|}
\hline Variable & No. (\%) \\
\hline Weight category according to gestational age & \\
\hline Appropriate & $98(90.7)$ \\
\hline Small & $7(6.5)$ \\
\hline Large & $3(2.8)$ \\
\hline Male gender & $51(47.2)$ \\
\hline
\end{tabular}

ultrasonography of the abdomen for diagnosis, which showed intraperitoneal free fluid. Two neonates underwent emergency surgical abdominal paracentesis, while one neonate was managed conservatively. The neonate born at 24 weeks gestation eventually died from an unrelated cause.

There were $2(1.9 \%)$ neonates with UVC-related venous thrombosis. Both patients were premature at 28 weeks and 31 weeks, with birth weights of 1,685 g and 1,630 g, respectively. Both neonates had UVCs inserted on Day 1 of life, which were noted to be malpositioned into the liver. Total parenteral nutrition was infused through both UVCs. The patients developed abdominal distension from Days 3-4 of life. The first neonate underwent abdominal ultrasonography, which showed umbilical thrombus extending to the left portal vein. Enteral feeds were withheld for this neonate and the thrombus was managed conservatively. However, the infant developed gastric perforation three days later, requiring surgery. The second neonate with UVC-related venous thrombosis presented with pneumoperitoneum and was found to have $30 \mathrm{~cm}$ of ischaemic bowel on laparotomy. Histopathology showed mesenteric vein thrombosis, but subsequent Doppler ultrasonography was normal and the thrombosis did not require further management.

\section{DISCUSSION}

The incidence of new-onset or worsening sepsis within 72 hours of the removal of UVCs in our unit was $14.8 \%(16 / 108)$. The umbilical CABSI rate in our study was 7 per 1,000 catheter days $(4.6 \%)$. However, if the more stringent United States Centers for Disease Control and Prevention criteria for CRBSI were used, this would have been only 2.8 per 1,000 catheter days (1.8\%). ${ }^{(10)}$

The CABSI rate in our study was comparable with that of other international studies. A 2002 Canadian Neonatal Network study yielded an umbilical CABSI rate of 7.2 per 1,000 catheter days. ${ }^{(11)}$ Another study from China in 2012 yielded a rate of 13.6 per 1,000 umbilical catheter days. ${ }^{(12)}$ The Canadian Neonatal Network UVCassociated CABSI rate seemed to have stayed stable at around 7.8-8.2 per 1,000 catheter days in another review published in $2015,{ }^{(9)}$ as part of a comparison with the peripherally inserted central catheter (PICC) line.

Clinical presentation of CABSI in neonates are varied and nonspecific. Presentation may include fever, respiratory symptoms, erythema or purulent discharge at the insertion site, and feed intolerance. ${ }^{(13)}$ Clinicians will generally remove the UVC in the context of symptomatic bacteraemia. A cohort study that evaluated how often neonates with CONS bacteraemia can be treated successfully without removing the central venous catheter showed 
Table II. Details of neonates with catheter-associated bloodstream infection (CABSI).

\begin{tabular}{|c|c|c|c|c|c|c|}
\hline $\begin{array}{l}\text { Gestational } \\
\text { age (wk); birth } \\
\text { weight (g) }\end{array}$ & $\begin{array}{l}\text { Age (day); } \\
\text { duration of } \\
\text { UVC use (day) }\end{array}$ & UVC position & $\begin{array}{l}\text { Blood } \\
\text { culture }\end{array}$ & Other cultures & Clinical presentation & Outcome \\
\hline $23+6 ; 685$ & $8 ; 8$ & Ideal & E. cloacae & $\begin{array}{l}\text { UAC/UVC tip: } \\
\text { E. cloacae }\end{array}$ & $\begin{array}{l}\text { Raised immature-to-total } \\
\text { neutrophil ratio, abdominal } \\
\text { fullness; work-up showed } \\
\text { sealed perforation with } \\
\text { meconium soiling }\end{array}$ & $\begin{array}{l}\text { Died on Day } 14 \\
\text { from sepsis }\end{array}$ \\
\hline $25+5 ; 860$ & $9 ; 9$ & $\begin{array}{l}\text { UVC \#1: deep } \\
\text { (Days 1-4), noted } \\
\text { leaking from tube } \\
\text { UVC \#2: } \\
\text { malpositioned }\end{array}$ & CONS & UVC tip: CONS & $\begin{array}{l}\text { Increased lability and } \\
\text { desaturations; treated as for } \\
\text { CABSI }\end{array}$ & Sepsis, treated \\
\hline $25+5 ; 740$ & $7 ; 6$ & Ideal & CONS & $\begin{array}{l}\text { Surface swabs: } \\
\text { Enterococcus faecalis } \\
\text { Sputum c/s: } \\
\text { E. aerogenes } \\
\text { Wound c/s: CONS }\end{array}$ & $\begin{array}{l}\text { High C-reactive protein; } \\
\text { work-up showed } \\
\text { spontaneous intestinal } \\
\text { perforation }\end{array}$ & $\begin{array}{l}\text { Underwent } \\
\text { surgery, well } \\
\text { at the time of } \\
\text { study }\end{array}$ \\
\hline $35 ; 1,415$ & $5 ; 4$ & Malpositioned & CONS & $\begin{array}{l}\text { UVC tip not sent; } \\
\text { cerebrospinal fluid/ } \\
\text { urine/stool cultures } \\
\text { negative }\end{array}$ & $\begin{array}{l}\text { Abdominal distension; } \\
\text { treated as for septic ileus }\end{array}$ & $\begin{array}{l}\text { Feeds resumed } \\
\text { after gut rest } \\
\times 48 \mathrm{hr}\end{array}$ \\
\hline $23+6 ; 535$ & $10 ; 14$ & Ideal & CONS & $\begin{array}{l}\text { ETT: E. cloacae, } \\
\text { Mycoplasma } \\
\text { hominis, } \\
\text { Ureaplasma } \\
\text { UAC/UVC tip: CONS }\end{array}$ & $\begin{array}{l}\text { Presented with hypotension; } \\
\text { work-up showed raised } \\
\text { infection markers; treated as } \\
\text { for CABSI }\end{array}$ & Sepsis, treated \\
\hline
\end{tabular}

CONS: coagulase-negative Staphylococcus; c/s: culture and sensitivity; E. aerogenes: Enterobacter aerogenes; E. cloacae: Enterobacter cloacae; ETT: endotracheal tube; UAC: umbilical arterial catheter; UVC: umbilical venous catheter

that retention of central venous catheters was successful in $46 \%$ of neonates with CONS bacteraemia but was never successful if the bacteraemia lasted for more four days. ${ }^{(14)}$ However, our practice was still more commonly to remove a central catheter if there was definite bacteraemia in a neonate.

It is important to consider whether the avoidance of UVC and its replacement with peripheral intravenous access and subsequent conversion to PICC would lead to better outcomes. Most of the current literature consists of retrospective cohort studies that have shown no additional advantage of UVC over PICC lines, whether in terms of success of insertion, complications or unplanned removal rates. Indeed, complication rates were similar between the two in many studies, ${ }^{(9,15,16)}$ while one multicentre retrospective cohort study by Shalabi et $\mathrm{al}^{(9)}$ found lower late-onset sepsis rates among infants who received only UVCs. Two randomised controlled trials have also shown similar complications rates for UVC and PICC lines. ${ }^{(17,18)}$

Evidence supporting earlier planned removal of UVCs to reduce $\mathrm{CABSI}$ rates is conflicting. A retrospective cohort study that evaluated the outcome of introducing a standardised PICC and care bundle showed that infants with UVC lasting over seven days had a greater risk of central line-associated bloodstream infection (odds ratio 5.48) than those with UVC lasting seven days or less. ${ }^{(19)}$ However, a recent Cochrane review by Gordon et al, ${ }^{(3)}$ which investigated early planned removal of UVCs within two weeks to prevent infection, included only one study - that by
O'Hara et al. ${ }^{(12-14)}$ It found no difference in CABSI rates (relative risk [RR] 0.65, 95\% confidence interval [Cl] 0.35-1.22), hospital mortality (RR 1.12, 95\% Cl 0.42-2.98) and catheter-associated thrombus necessitating removal (RR 0.33, 95\% Cl 0.01-7.94). More infants in the early planned removal group than the expectant management group required percutaneous insertion of a central catheter (or PICC). These studies, which compared data of infants with UVC duration of seven days versus 14 days, suggest that there is a paucity of published literature on whether two weeks is too long a duration for UVC insertion.

While there are various methods to define an ideal UVC position, (20) the cardiac silhouette method is reported to be superior to the vertebral level method for predicting the ideal venous catheter tip position. ${ }^{(21)}$ The diaphragm method gives a better correlation to the cardiac silhouette on radiological imaging and hence, was used as the method to determine ideal UVC positioning in our study.

Malpositioned UVCs lead to a higher risk of complications when administering hypertonic solution through these catheters and should be avoided. Published case series have reported severe hepatic injuries associated with UVCs that were malpositioned into the hepatic portal circulation and low-lying UVCs. ${ }^{(5)}$ Extravasation injuries were also associated with hypertonic solutions running through low-lying catheters, where blood flow is slower, predisposing to this complication. ${ }^{(22)}$ Among the three neonates who had extravasation in our study, two had low-lying UVCs and one had a malpositioned UVC. 
Risk factors for neonatal venous thrombosis include extreme prematurity (gestational age $\leq 27.7$ weeks), extremely low birth weight (birth weight $<900 \mathrm{~g}$ ), raised haematocrit levels above $55 \%$, and increased duration of indwelling central venous catheters and malpositioned UVCs. ${ }^{(4,23)}$ In one prospective cohort study, necrotising enterocolitis was associated with unrecognised withdrawal of UVC into the portal vein or ductus venosus, ${ }^{(24)}$ while a case of intestinal perforation secondary to UVC passing through an anomalous patent vitellointestinal duct has also been reported. ${ }^{(25)}$ These two studies demonstrate that the compromise of vascular supply in malpositioned UVCs, with or without thrombosis, may cause gastrointestinal perforations. Similarly, the two neonates with UVC-related gastrointestinal perforation in our study had UVC malpositioning and thrombosis, suggesting that vascular compromise may be a possible pathophysiology.

There is currently great heterogeneity of practice within and between neonatal units, and review of clinical practice, such as the present study, would greatly help in risk assessment and to refine indications for use of UVCs in neonates. Among the many areas of controversies are the indications for placement in terms of gestational age or birth weight, duration of UVC insertion, how to manage low-lying UVCs and even whether to repeat radiography after the repositioning of UVCs. In a 2014 survey from the United Kingdom of up to 72 neonatal units, ${ }^{(8)} 52 \%$ of units kept the UVCs for $\leq 7$ days, $24 \%$ of units for $8-10$ days, $21 \%$ of units for 14 days and $3 \%$ of units had no upper limit. These authors found that most respondent units accepted low-lying UVCs for either routine use (44\%) or limited use (48\%). Of these units, $76 \%$ removed low-lying UVCs electively within seven days (with approximately two-thirds removing them within three days) and $11 \%$ by 14 days, while only $3 \%$ of units placed no maximum time limit for the removal of low-lying UVCs.

Venous catheter extravasation was more prevalent than previously recognised and most cases involved low-lying UVCs. ${ }^{(22)}$ Towards this end, the British Association for Perinatal Medicine issued guidelines in 2015 after literature review and consultation with practitioners incorporating the following three main practice points: (a) any clinical deterioration of an infant in whom a central venous catheter is present should raise the question of catheter-related complications, particularly infection, extravasation and tamponade; (b) all central catheter tips should be positioned outside the cardiac silhouette; and (c) a UVC tip should ideally be sited at T8-T9 (assuming this lies outside the cardiac silhouette). A UVC tip sited at or below T10 carries a significantly higher risk of extravasation. It may be necessary to use these catheters in the short term, but they should be replaced at the earliest opportunity. ${ }^{(26)}$

Similarly, our study found that UVCs, although an important means of vascular access for neonates, had a high rate of complications and should be used judiciously. However, the alternative of PICC lines was equally risky. Guidelines should be developed so that the use of central line is judicious, and there is evidence that such guidelines can reduce the use of UVCs. ${ }^{(27)}$ In one such quality improvement project, UVCs were generally recommended only for infants of gestational age 28 weeks or less. We would suggest for individual neonatal units to develop guidelines for UVC use and more importantly, to audit their complication rates. In addition, we suggest that UVCs be kept generally to a maximum of seven days' duration, unless under extenuating circumstances, and that malpositioned UVCs should be removed. Low-lying UVCs should be removed early, probably within 48 hours.

In conclusion, our incidence of CABSI was comparable to that of other centres. UVC-associated extravasation was more common than previously thought and was associated with both malpositioned and low-lying UVCs. Judicious use of UVCs in neonates is required to reduce the complication rates associated with them. Low-lying UVCs should be used with caution, as neonates are at risk of severe morbidities such as total parenteral nutrition extravasation or venous thrombosis. The presentation of CABSI or neonatal venous thrombosis is heterogeneous and may be subtle. A high index of suspicion is required for the further evaluation and management of neonates with UVCs.

\section{REFERENCES}

1. Nash P. Umbilical catheters, placement, and complication management. J Infus Nurs 2006; 29:346-52.

2. Cronin WA, Germanson TP, Donowitz LG. Intravascular catheter colonization and related bloodstream infection in critically ill neonates. Infect Control Hosp Epidemiol 1990; 11:301-8.

3. Gordon A, Greenhalgh M, McGuire W. Early planned removal of umbilical venous catheters to prevent infection in newborn infants. Cochrane Database Syst Rev 2017; 10:CD012142.

4. Park CK, Paes BA, Nagel K, Chan AK, Murthy P; Thrombosis and Hemostasis in Newborns (THiN) Group. Neonatal central venous catheter thrombosis: diagnosis, management and outcome. Blood Coagul Fibrinolysis 2014; 25:97-106.

5. Grizelj R, Vukovic J, Bojanic K, et al. Severe liver injury while using umbilical venous catheter: case series and literature review. Am J Perinatol 2014; 31:965-74.

6. Abiramalatha T, Kumar M, Shabeer MP, Thomas N. Advantages of being diligent: lessons learnt from umbilical venous catheterisation in neonates. BMJ Case Rep 2016; pii:bcr2015214073.

7. Kurtom W, Quast D, Worley L, Oelberg DG. Incorrect umbilical vein catheterization is associated with severe periventricular hemorrhages and mortality in extremely premature newborns. J Neonatal Perinatal Med 2016; 9:67-72.

8. Hollingsworth C, Clarke P, Sharma A, Upton M. National survey of umbilical venous catheterisation practices in the wake of two deaths. Arch Dis Child Fetal Neonatal Ed 2015; 100:F371-2.

9. Shalabi M, Adel M, Yoon E, et al; Canadian Neonatal Network. Risk of infection using peripherally inserted central and umbilical catheters in preterm neonates. Pediatrics 2015; 136:1073-9.

10. Tomlinson D, Mermel LA, Ethier MC, et al. Defining bloodstream infections related to central venous catheters in patients with cancer: a systematic review. Clin Infect Dis 2011; 53:697-710.

11. Chien LY, Macnab Y, Aziz K, et al; Canadian Neonatal Network. Variations in central venous catheter-related infection risks among Canadian neonatal intensive care units. Pediatr Infect Dis J 2002; 21:505-11.

12. Hei MY, Zhang XC, Gao XY, et al. Catheter-related infection and pathogens of umbilical venous catheterization in a neonatal intensive care unit in China. Am J Perinatol 2012; 29:107-14.

13. Fallat ME, Gallinaro RN, Stover BH, Wilkerson S, Goldsmith LJ. Central venous catheter bloodstream infections in the neonatal intensive care unit. J Pediatr Surg 1998; 33:1383-7.

14. Karlowicz MG, Furigay PJ, Croitoru DP, Buescher ES. Central venous catheter removal versus in situ treatment in neonates with coagulase-negative staphylococcal bacteremia. Pediatr Infect Dis J 2002; 21:22-7.

15. Arnts IJ, Bullens LM, Groenewoud JM, Liem KD. Comparison of complication rates between umbilical and peripherally inserted central venous catheters in newborns. J Obstet Gynecol Neonatal Nurs 2014; 43:205-15.

16. Mutlu M, Aslan Y, Kul S, Yılmaz G. Umbilical venous catheter complications in newborns: a 6-year single-center experience. J Matern Fetal Neonatal Med 2016; 29:2817-22.

17. Dongara AR, Patel DV, Nimbalkar SM, Potana N, Nimbalkar AS. Umbilical venous catheter versus peripherally inserted central catheter in neonates: a randomized controlled trial. J Trop Pediatr 2017; 63:374-9. 
18. Butler-O'Hara M, Buzzard CJ, Reubens L, et al. A randomized trial comparing long-term and short-term use of umbilical venous catheters in premature infants with birth weights of less than 1251 grams. Pediatrics 2006; 118:e25-35.

19. Butler-O'Hara M, D'Angio CT, Hoey H, Stevens TP. An evidence-based cathete bundle alters central venous catheter strategy in newborn infants. J Pediatr 2012; 972-7.e2.

20. Guimarães AF, Souza AA, Bouzada MC, Meira ZM. Accuracy of chest radiography for positioning of the umbilical venous catheter. J Pediatr (Rio J) 2017; 93:172-8.

21. Hoellering AB, Koorts PJ, Cartwright DW, Davies MW. Determination of umbilical venous catheter tip position with radiograph. Pediatr Crit Care Med 2014; 15:56-61.

22. Saboo A, Shama A, Edelman J, Salter C. PC.112: Umbilical venous catheter extravasation: a retrospective study and quality improvement programme. Poster presentation. Arch Dis Child Fetal Neonatal Ed 2014; 99:A75.
23. Narang S, Roy J, Stevens TP, et al. Risk factors for umbilical venous catheter-associated thrombosis in very low birth weight infants. Pediatr Blood Cancer 2009; 52:75-9.

24. Sulemanji M, Vakili K, Zurakowski D, et al. Umbilical venous catheter malposition is associated with necrotizing enterocolitis in premature infants. Neonatology 2017; 111:337-43.

25. Marshall AS, Jayapal SS, Whitburn JA, Akinbiyi BA, Willetts IE. Recognising serious umbilical cord anomalies. BMJ Case Rep 2013; pii:bcr2013201663.

26. British Association of Perinatal Medicine. Use of central venous catheters in neonates: a framework for practice. Available at: https://hubble-live-assets. s3.amazonaws.com/bapm/attachment/file/17/BAPM_CVC_final_Jan16_ addition_Aug_2018_.pdf. Accessed January 11, 2021.

27. Shahid S, Dutta S, Symington A, Shivananda S; McMaster University NICU. Standardizing umbilical catheter usage in preterm infants. Pediatrics 2014; 133:e1742-52. 